\title{
Analysis of Prognostic Factors in Patients with Transitional Cell Carcinoma of the Bladder Treated with Radical Cystectomy
}

\author{
Alberto A. Antunes, Luciano J. Nesrallah, Marcos F. Dall'Oglio, Yuri A. Ferreira, Carlo C. \\ Passerotti, Katia R. Leite, Valdemar Ortiz, Miguel Srougi \\ Department of Urology, University of Sao Paulo (USP), Department of Urology, Beneficencia \\ Portuguesa Hospital, Sao Paulo, and Laboratory of Surgical and Molecular Pathology, Syrian \\ Lebanese Hospital, Sao Paulo, Brazil
}

\begin{abstract}
Objective: To analyze the results of the treatment of transitional cell carcinoma (TCC) of the bladder with radical cystectomy and determine which prognostic factors can be utilized as disease-free survival and cancer-specific survival independent variables.

Materials and Methods: Medical records of 113 patients submitted to radical cystectomy and bilateral iliac lymphadenectomy between 1993 and 2005 were reviewed. The risk factors analyzed were age, sex, pathological stage, tumor grade, presence of carcinoma in situ and the presence of lymph nodes involvement.

Results: After a mean follow-up of $31.7 \pm 28.5$ months, 46 patients $(40.7 \%)$ presented recurrence and 24 patients $(21.2 \%)$ died due to cancer. Only pathological stage and the lymph nodes involvement became independent variables for recurrence and survival. Patients with T4 stage presented 9.6 times the risk of recurrence of the disease when compared with stage T0 patients $(\mathrm{p}=0.010)$ and the patients with lymph node involvement presented 2.5 times the risk of recurrence $(p=0.047)$ and 3.1 times the risk of death $(\mathrm{p}=0.022)$ when compared to patients without lymph nodes involvement.

Conclusions: Pathological stage and the involvement of lymph nodes represented more important prognostic variables, and in the presence of advanced stage tumors (T3/T4) and involvement of lymph nodes, the institution of adjuvant treatment should be considered.
\end{abstract}

Key words: bladder neoplasms; transitional cell; cystectomy; prognosis Int Braz J Urol. 2006; 32: 35-42

\section{INTRODUCTION}

Bladder cancer is responsible for $7 \%$ of all types of neoplasia in men and $2 \%$ in women (1). Around $25 \%$ of the diagnosed cases are infiltrative tumors, and presently radical cystectomy is the treatment of choice in such cases (2). However, clinical evolution of these patients is not uniform and, despite aggressive surgical treatment, around $50 \%$ die after 5 years of follow-up (3-5).
Such high treatment failure rates can be explained by the fact that bladder infiltrative tumors present a trend towards the development of metastasis, which was not identified by diagnostic methods available at the time of treatment $(6,7)$. For this reason, even though its benefit is not clearly defined, adjuvant chemotherapy has been indicated as a strategy to improve treatment efficacy (6).

Two important measures that can contribute to improve therapeutic results are the development 
of new chemotherapy agents, and an adequate selection of the patients who are candidates for adjuvant treatment $(8,9)$. In this context, the definitions of recurrence and survival independent prognostic factors that allow the selection of high-risk patients that can benefit from adjuvant treatment assume a fundamental importance. Previous studies have demonstrated that pathological stage, the tumor grade, the presence of carcinoma in situ (CIS) and the involvement of lymph nodes constitute important prognostic factors in patients with bladder TCC $(3,10)$.

The objective of the present study is to analyze the results of bladder TCC treatment with radical cystectomy and determine which prognostic factors can be used as mortality and recidivation independent variables due to this aggressive behavior neoplasia.

\section{MATERIALS AND METHODS}

Between March 1993 and January 2005, the medical records of 153 patients diagnosed with bladder cancer submitted to radical cystectomy and obturatory lymphadenectomy were revised. After the exclusion of patients that received neoadjuvant chemotherapy, that presented tumors of other histology types and those who had incomplete medical records and did not have available follow-up, the final group was composed of 113 patients.

Preoperative diagnosis was performed by transurethral resection. All patients were staged with thorax radiography and computerized tomography of the abdomen and pelvis, and were considered bearers of a localized disease. The indications of radical cystectomy included the presence of tumors compromising the muscular layer (T2), and superficial tumors refractory to multifocal or intravesical therapy. Fifteen patients with extravesical disease (T3-T4 or positive lymph nodes) were submitted to adjuvant chemotherapy with metotrexate, vinblastine, adriamycin and cisplatin (M-VAC).

Risk factors analyzed were age, sex, pathological stage, tumor grade, presence of CIS and an indication of involvement of the lymph nodes. The analysis of the pathological stage and tumor grade was conducted using the TNM system developed in 1997 and WHO respectively $(11,12)$. For the analysis of the pathological stage we utilized the T0, T1 (T1 + Tis), T2 (T2a + T2b), T3 (T3a + T3b) and T4 categories. Grade 1 and 2 tumors were considered low grade, while grade 3 and 4 tumors were considered high grade.

Table-1 describes the characteristics of the 113 patients studied. The mean age was 65.9 years (42 to 90 ), and the majority of patients $(85.8 \%$ ) were male. As for the stage, we observed that $39 \%$ of the patients presented extravesical disease (T3 and T4) and the large majority (86.9\%) presented high-grade tumors ( 3 and 4 ). The presence of CIS was observed in $40.7 \%$ of the patients, and the involvement of lymph nodes in $15.7 \%$. Twenty nine patients did not have

Table 1 - Characteristics of patients studied.

\begin{tabular}{ll}
\hline & $\mathbf{N}(\%)$ \\
\hline $\begin{array}{l}\text { Age } \\
\text { (mean / median) } \\
\text { (variation) }\end{array}$ & $65.9 \pm 9.7 / 67.0$ \\
Sex & 42 a 90 \\
(male / female) & \\
& \\
Pathological stage & $97(85.8) / 16(14.2)$ \\
T0 & \\
Tis & \\
T1 & $13(11.5)$ \\
T2a & $9(8.0)$ \\
T2b & $18(15.7)$ \\
T3a & $18(15.7)$ \\
T3b & $11(9.7)$ \\
T4 & $12(10.6)$ \\
Grade & $23(20.4)$ \\
Low & $9(8.0)$ \\
High & \\
Carcinoma in situ & \\
(yes / no) & $11(13.1)$ \\
Lymph nodes involvement & $73(86.9)$ \\
(yes / no) & \\
\hline
\end{tabular}


information regarding the tumor grade while 43 lacked information regarding lymph node involvement. Mean postoperative follow-up was $31.7 \pm 28.5$ months (median 24, 1 to 138).

During the postoperative period, follow-up consisted of a visit 2 months after the surgery and every 4 months until 1 year was completed. After this period, patients were seen every 6 months or less in cases of progression or death. On every visit, patients were submitted to clinical exams, thorax radiography and computerized tomography of the abdomen and pelvis. Recurrence was defined as: a) local = presence of a new tumor lesion in the pelvis and/or retroperitoneum or abdominal wall; b) systemic $=$ in other parts of the body; and c) urothelial = when present in the urethra or upper urinary tract.

The main variables studied were disease-free survival and cancer-specific survival. Patients that died due to other causes were considered as censures and were followed until the date of death. The KaplanMeier method was utilized to determine survival curves and statistical significance assessed by LogRank and Breslow tests. Multivariate analysis was performed with the Cox regression model showing a confidence interval (CI) of 95\%. The value of $\mathrm{p}<0.05$ was considered statistically significant.

\section{RESULTS}

Forty-six patients $(40.7 \%)$ presented recurrence; 20 local (17.7\%), 25 systemic $(22.1 \%)$ and 5 urothelial $(4.4 \%)$. The most frequent systemic metastasis sites were bone (24\%), lung (22\%) and liver $(13 \%)$. Four patients presented more than one type of recurrence simultaneously - 3 local and systemic and 1 systemic and urothelial. The mean disease-free survival rate was estimated at 69 months (KaplanMeier, CI 95\% [55 to 83]). Twenty four patients (21.2\%) died due to cancer. The mean time of cancer-specific survival was estimated at 94 months (Kaplan-Meier, CI 95\% [79 to 109]).

Through the Kaplan-Meier method, we observed that both pathological stage and the presence of lymph node involvement significantly influenced disease-free and cancer-specific survival. Of patients with stage $\mathrm{T} 0, \mathrm{~T} 1, \mathrm{~T} 2, \mathrm{~T} 3$, and $\mathrm{T} 4$, recurrences were observed in $7.3 \%, 33.3 \%, 34.5 \%, 62.9 \%$ and $44.4 \%$ of patients respectively (Breslow, $\mathrm{p}=0.009$ ), and death in $0 \%, 18.5 \%, 9.3 \%, 37.1 \%$ and $33.3 \%$ respectively (Breslow, $\mathrm{p}=0.002$ ) (Figures-1 and 2). As for the analysis of lymph nodes, recurrences occurred in 8 of 11 patients with compromised lymph nodes (72.7\%) and in 20 of 59 patients without compromised lymph nodes (33.9\%) (Log-Rank, $\mathrm{p}=0.015)$, Figure-3. Death occurred in $6(54.5 \%)$ and $12(20.3 \%)$ patients of the 2 groups respectively (Log-Rank, $\mathrm{p}=$ 0.015) (Figure-4).

In the Cox regression analysis, only pathological stage and a compromise in the lymph nodes constituted recurrence independent variables in uni- and multi-variate analysis (Tables-2 and 3). Even though patients with high grade tumors presented 3.4 times the risk of recurrence when compared to patients with low grade tumors, this result showed to be only marginally significant $(\mathrm{p}=0.093)$. According to Table-3, we observed that patients with stage $\mathrm{T} 4$ presented a recurrence risk 9.6 times greater than patients with stage $\mathrm{T} 0(\mathrm{p}=0.010)$, and patients presenting involvement of the lymph nodes presented a recurrence risk 2.5 times greater than patients without compromised lymph nodes $(p=0.047)$. Similar results were observed in relation to cancer-specific survival (Table-4). Patients with lymph node involvement presented a death risk 3.1 times greater when compared to those without lymph node involvement $(\mathrm{p}=0.022)$. The inclusion of stage in this model was not possible since no patient with stage $\mathrm{T} 0$ died due to the disease.

\section{COMMENTS}

In the present study, we analyzed the results of the treatment of 113 patients with diagnosis of bladder TCC and determined which variables could be used as independent prognostic factors of disease-free and cancer-specific survival. Despite the radical surgical treatment, after a mean follow-up of 32 months, around $40 \%$ of the patients presented a recurrence and $21 \%$ died of cancer. The only prognostic factors independent from disease-free survival were pathological stage and lymph node involvement. As for the 


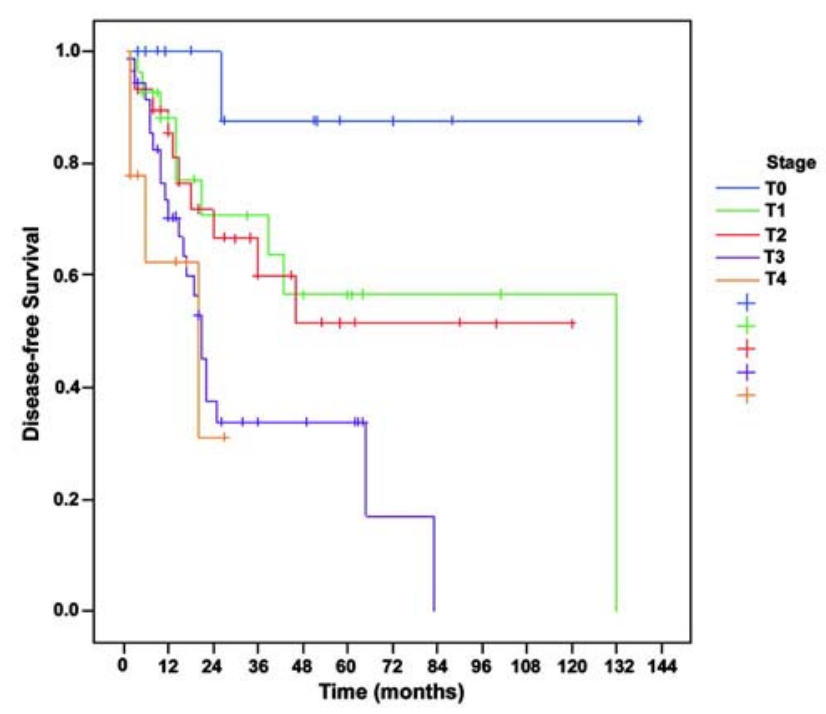

Figure 1-Disease-free survival according to pathological stage (Breslow, $p=0.009$ ).

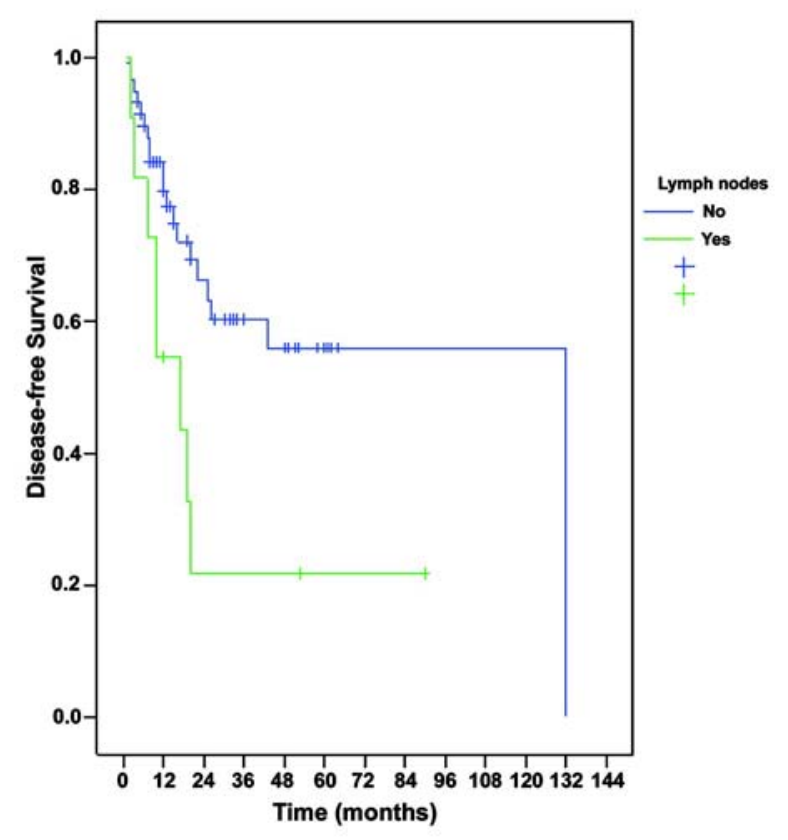

Figure 3 - Disease-free survival according to lymph node involvement (Log-Rank, $p=0.015)$.

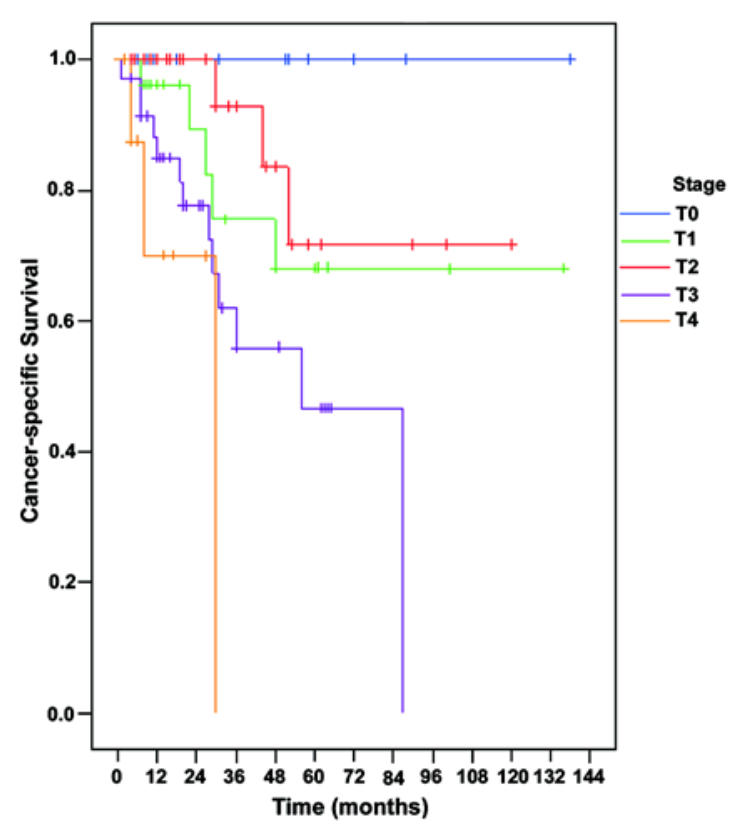

Figure 2 - Cancer-specific survival according to pathological stage (Breslow; $p=0.002$ ).

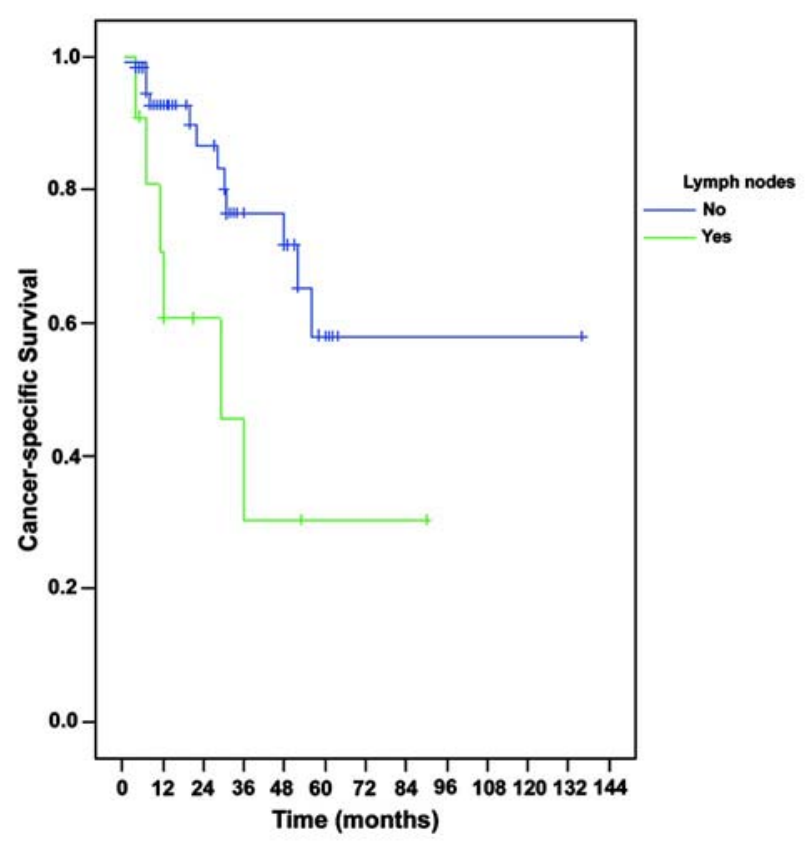

Figure 4 - Cancer-specific survival according to lymph node involvement (Log-Rank; $p=0.015$ ). 
Table 2 - Results of Cox regression to determine the association of variables with disease-free survival (univariate analysis).

\begin{tabular}{|c|c|c|c|}
\hline & Relative Risk & CI at $95 \%$ & p Value \\
\hline \multicolumn{4}{|l|}{ Univariate analysis } \\
\hline $\begin{array}{l}\text { Age } \\
(\geq 67 \text { years } /<67 \text { years })\end{array}$ & 1.03 & {$[0.57-1.87]$} & 0.910 \\
\hline $\begin{array}{l}\text { Sex } \\
(\text { male / female })\end{array}$ & 1.64 & {$[0.65-4.16]$} & 0.299 \\
\hline $\begin{array}{l}\text { Stage } \\
\text { T1/T0 } \\
\text { T2/T0 } \\
\text { T3/T0 } \\
\text { T4/T0 }\end{array}$ & $\begin{array}{r}5.91 \\
6.37 \\
14.01 \\
19.04\end{array}$ & $\begin{array}{l}{[0.75-46.80]} \\
{[0.80-50.94]} \\
{[1.84-106.71]} \\
{[2.05-176.48]}\end{array}$ & $\begin{array}{l}0.011 \\
0.093 \\
0.081 \\
0.011 \\
0.010\end{array}$ \\
\hline $\begin{array}{l}\text { Grade } \\
\text { (Low / High) }\end{array}$ & 3.40 & {$[0.82-14.17]$} & 0.093 \\
\hline $\begin{array}{l}\text { Ca in situ } \\
\text { (yes / no) }\end{array}$ & 1.36 & {$[0.75-2.45]$} & 0.311 \\
\hline $\begin{array}{l}\text { Lymph nodes } \\
\text { (yes / no) }\end{array}$ & 2.68 & {$[1.17-6.14]$} & 0.020 \\
\hline
\end{tabular}

Table 3 - Results of Cox regression to determine the association of variables to disease-free survival (multivariate analysis).

Relative Risk $\quad$ CI at $95 \% \quad$ p Value

\section{Multivariate analysis}

\section{Stage}

$\mathrm{T} 1 / \mathrm{T} 0$

T2/T0

T3/T0

$\mathrm{T} 4 / \mathrm{T} 0$

\section{Lymph nodes}

(yes / no)

1.34
1.46
4.05
9.60

2.51

$\begin{array}{ll} & 0.024 \\ {[0.14-12.86]} & 0.093 \\ {[0.16-13.71]} & 0.081 \\ {[0.52-31.79]} & 0.011 \\ {[1.06-87.04]} & 0.010\end{array}$

0.024

0.081

0.011

010

cancer-specific survival, lymph node involvement was also an independent variable. Even though the inclusion of pathological stage in the regression model for mortality was not possible, we observed that while no patient with stage T0 died, death occurred in $36 \%$ of the cases when the stage was T3/T4. 
Prognostic Factors in Carcinoma of the Bladder

Table 4-Cox regression model to determine the association of variables to cancer-specific survival (univariate analysis).

\begin{tabular}{|c|c|c|c|}
\hline & Relative Risk & CI at $95 \%$ & p Value \\
\hline \multicolumn{4}{|l|}{ Univariate analysis } \\
\hline \multicolumn{4}{|l|}{ Age } \\
\hline$(\geq 67$ years $/<67$ years $)$ & 1.42 & {$[0.62-3.25]$} & 0.405 \\
\hline \multicolumn{4}{|l|}{ Sex } \\
\hline (male / female) & 2.41 & {$[0.57-10.25]$} & 0.235 \\
\hline \multicolumn{4}{|l|}{ Grade } \\
\hline (high / low) & 3.02 & {$[0.40-22.77]$} & 0.283 \\
\hline \multicolumn{4}{|l|}{ Ca in situ } \\
\hline (yes / no) & 1.33 & {$[0.59-2.99]$} & 0.486 \\
\hline \multicolumn{4}{|l|}{ Lymph nodes } \\
\hline (yes / no) & 3.17 & {$[1.19-8.46]$} & 0.022 \\
\hline
\end{tabular}

Many studies analyzed the prognostic factors in patients with bladder TCC treated with radical cystectomy. The pathological stage and lymph nodes involvement have been considered by many authors to be the main prognostic factors in these patients $(3,4,13,14)$. In an analysis of 130 patients, Soloway et al. (4) observed that 5-year survival rates were $82 \%$ for patients with superficial tumors, $65 \%$ for those with T2 and 28\% for patients with T3/T4, while patients with lymph node involvement presented a 5-year survival rate of $18 \%$ against $65 \%$ for patients presenting no lymph node involvement. Similarly, in an analysis of 369 patients treated with radical cystectomy as monotherapy, Bassi et al. (13) demonstrated that while in univariate analysis of pathological stage, the involvement of lymph nodes, the presence of microvascular and perineural invasion and the presence of obstruction in upper urinary tract were determinant of global survival, in multivariate analysis only the first two were independent variables. More recently, an analysis of 114 patients considering the variables of age, sex, pathological stage, grade, lymph node involvement, lymphatic invasion, vascular invasion and the presence of CIS found that only pathological stage and lymph node involvement were independent factors of recurrence (14).

On the other hand, many authors have highlighted the prognostic value of other variables. In an analysis of 218 patients, Cheng et al. (2) found the tumor size, pathological stage and involvement of lymph nodes were independent variables for metastasis-free, cancer-specific and global survival rates. The presence of positive margins was independently associated with recurrence-free and cancer-specific survival, and age, lymph node involvement and the presence of positive margins was associated with local recurrence-free survival. Later, in analyzing 64 patients with stage T2 only, the same authors demonstrated that only tumor size and involvement of the lymph nodes were significantly associated with disease free and cancer-specific survival, and they proposed a change in the sub-classification of $\mathrm{T} 2$ stage based on tumor size 1 (15). On the other hand, in an analysis of 283 patients, Leissner et al. (16) demonstrated that the presence of blood vessel invasion together with stage and lymph node involvement were the sole determinant variables for disease-free survival. The value of microvascular invasion was also highlighted by Hong et al. (7) in a retrospective analy- 
sis of 125 patients, demonstrating that only this and pathological stage were independent variables for global survival - the former being more important than the latter. Patients with lymph nodes involvement, however, were not included in the analysis.

In the present study, age, sex, tumor grade and the presence of carcinoma in situ did not constitute important prognostic factors. While the majority of studies demonstrate that sex does not present any prognostic value $(2,3,5,10,15,16)$, the results regarding age analysis are more conflicting. Some point out that age can be determinant of recurrence $(2,15)$, cancer-specific survival (10) and global survival (15), while others demonstrate not to have any prognostic value at all $(3,5,7,14,16)$. Our results correspond to the latter studies and thus suggest that radical cystectomy can be indicated for older patients as a form of treatment for bladder TCC. The presence of CIS has also showed conflicting results. Even though the presence of CIS is traditionally related to a poor prognosis, many studies do not reproduce these results $(5,14)$, or even show that patients with CIS present better cancer-specific survival and global survival rates when treated with radical cystectomy $(2,10)$. As for the grade, the fact that the majority of bladder infiltrated tumors present a high grade $(87 \%$ of cases in the present series) can, in part, explain why tumor grade presents a low prognostic value in these patients. In fact, many authors confirm these findings $(2,3,5,7)$. In the present study, even though patients with high grade tumors have presented around 3 times the risk of recurrence and mortality when compared to patients with low grade tumors, these results did not present statistical significance.

Despite aggressive treatment with radical cystectomy, 46 patients $(40.7 \%)$ presented recurrence and 24 patients $(21.2 \%)$ died due to cancer. One of the main factors responsible for the low rate of success with the curative surgical treatment of infiltrating bladder tumors is its predilection for hidden metastasis during treatment (7). This fact is clear when we observe that even the absence of residual tumors in the surgical specimen (T0) does not guarantee successful treatment. Frazier et al. (10) demonstrated that
$30 \%$ of their 75 patients with stage T0 died due to bladder tumors after 5 years. In our series, even though none of the 13 patients with T0 died, 1 of them (7.3\%) presented recurrence after 24 months of follow-up. It is possible that this number could increase if those patients are followed for a longer period.

A limitation of the present study is the fact that 15 patients received adjuvant chemotherapy. All those patients presented either stage T3/T4 or lymph node involvement. The recurrence and mortality rates of this group were $67 \%$ and $40 \%$ respectively, while $53 \%$ and $31 \%$ of the 32 patients T3/T4 that did not receive any adjuvant treatment presented recurrence and death respectively. These numbers suggest that chemotherapy did not represent benefits, however this analysis is restricted since patient selection for adjuvant treatment was not randomized.

Finally, we conclude that bladder infiltrating TCC represents an aggressive neoplasia with high rates of recurrence and mortality in spite of the radical surgery treatment. Pathological stage and lymph node involvement represent the most important variables for recurrence and survival, and in the presence of tumors with advanced stage (T3/T4) and involvement of lymph nodes, the institution of adjuvant treatment should be considered. However, these results should be analyzed carefully until other variables, such as tumor size, the presence of microvascular and perineural invasion, epidermoid differentiation and surgical margin compromise can be included in this study.

\section{ACKNOWLEDGEMENTS}

Adriana Sanudo made the statistical analysis.

\section{CONFLICT OF INTEREST}

None declared.

\section{REFERENCES}

1. Jemal A, Murray T, Ward E, Samuels A, Tiwari RC, Ghafoor A, et al.: Cancer statistics, 2005. CA Cancer J 
Clin. 2005; 55: 10-30. Erratum in: CA Cancer J Clin. 2005; 55: 259.

2. Cheng L, Weaver AL, Leibovich BC, Ramnani DM, Neumann RM, Scherer BG, et al.: Predicting the survival of bladder carcinoma patients treated with radical cystectomy. Cancer. 2000; 88: 2326-32.

3. Mazzucchelli L, Bacchi M, Studer UE, Markwalder R, Sonntag RW, Kraft R: Invasion depth is the most important prognostic factor for transitional-cell carcinoma in a prospective trial of radical cystectomy and adjuvant chemotherapy. Int J Cancer. 1994; 57: 15-20.

4. Soloway MS, Lopez AE, Patel J, Lu Y: Results of radical cystectomy for transitional cell carcinoma of the bladder and the effect of chemotherapy. Cancer. 1994; 73: 1926-31.

5. Knap MM, Lundbeck F, Overgaard J: Prognostic factors, pattern of recurrence and survival in a Danish bladder cancer cohort treated with radical cystectomy. Acta Oncol. 2003; 42: 160-8.

6. Skinner DG, Daniels JR, Russell CA, Lieskovsky G, Boyd SD, Nichols P, et al.: The role of adjuvant chemotherapy following cystectomy for invasive bladder cancer: a prospective comparative trial. J Urol. 1991; 145: 459-64; discussion 464-7.

7. Hong SK, Kwak C, Jeon HG, Lee E, Lee SE: Do vascular, lymphatic, and perineural invasion have prognostic implications for bladder cancer after radical cystectomy? Urology. 2005; 65: 697-702.

8. Bamias A, Deliveliotis Ch, Aravantinos G, Kalofonos Ch, Karayiannis A, Dimopoulos MA, et al.: Adjuvant chemotherapy with paclitaxel and carboplatin in patients with advanced bladder cancer: a study by the Hellenic Cooperative Oncology Group. J Urol. 2004; 171: 1467-70.

9. Yang MH, Yen CC, Chen PM, Wang WS, Chang YH, Huang WJ, et al.: Prognostic-factors-based risk-strati- fication model for invasive urothelial carcinoma of the urinary bladder in Taiwan. Urology. 2002; 59: 232-8; discussion 238-9.

10. Frazier HA, Robertson JE, Dodge RK, Paulson DF: The value of pathologic factors in predicting cancerspecific survival among patients treated with radical cystectomy for transitional cell carcinoma of the bladder and prostate. Cancer. 1993; 71: 3993-4001.

11. Sobin LH, Wittekind CH. International Union Against Cancer (UICC). Urinary Bladder, in TNM Classification of Malignant Tumors. New York, Wiley-Liss. 1997; pp 187-93.

12. Epstein JI, Amin MB, Reuter VR, Mostofi FK: The World Health Organization/International Society of Urological Pathology consensus classification of urothelial (transitional cell) neoplasms of the urinary bladder. Bladder Consensus Conference Committee. Am J Surg Pathol. 1998; 22: 1435-48.

13. Bassi P, Ferrante GD, Piazza N, Spinadin R, Carando R, Pappagallo G, et al.: Prognostic factors of outcome after radical cystectomy for bladder cancer: a retrospective study of a homogeneous patient cohort. J Urol. 1999; 161: 1494-7.

14. Harada K, Sakai I, Hara I, Eto H, Miyake H: Prognostic significance of vascular invasion in patients with bladder cancer who underwent radical cystectomy. Int J Urol. 2005; 12: 250-5.

15. Cheng L, Neumann RM, Scherer BG, Weaver AL, Leibovich BC, Nehra A, et al.: Tumor size predicts the survival of patients with pathologic stage T2 bladder carcinoma: a critical evaluation of the depth of muscle invasion. Cancer. 1999; 85: 2638-47.

16. Leissner J, Koeppen C, Wolf HK: Prognostic significance of vascular and perineural invasion in urothelial bladder cancer treated with radical cystectomy. J Urol. 2003; 169: 955-60.

\section{Correspondence address:}

Dr. Alberto A. Antunes

R. Dr. Diogo de Faria, 1201 / 58

São Paulo, SP, 04037-004, Brazil

E-mail: aantunes_uro@yahoo.com 